\title{
BKM-react, an integrated biochemical reaction database
}

\author{
Maren Lang ${ }^{\dagger}$, Michael Stelzer ${ }^{\dagger}$ and Dietmar Schomburg ${ }^{*}$
}

\begin{abstract}
Background: The systematic, complete and correct reconstruction of genome-scale metabolic networks or metabolic pathways is one of the most challenging tasks in systems biology research. An essential requirement is the access to the complete biochemical knowledge - especially on the biochemical reactions. This knowledge is extracted from the scientific literature and collected in biological databases. Since the available databases differ in the number of biochemical reactions and the annotation of the reactions, an integrated knowledge resource would be of great value.

Results: We developed a comprehensive non-redundant reaction database containing known enzyme-catalyzed and spontaneous reactions. Currently, it comprises 18,172 unique biochemical reactions. As source databases the biochemical databases BRENDA, KEGG, and MetaCyc were used. Reactions of these databases were matched and integrated by aligning substrates and products. For the latter a two-step comparison using their structures (via InCh/s) and names was performed. Each biochemical reaction given as a reaction equation occurring in at least one of the databases was included.

Conclusions: An integrated non-redundant reaction database has been developed and is made available to users. The database can significantly facilitate and accelerate the construction of accurate biochemical models.
\end{abstract}

\section{Background}

For the construction of cellular models, the development of organism-specific reaction networks is essential. A number of sources for biochemical reactions exist, as the databases BRENDA [1], KEGG [2], and MetaCyc [3]. In general, the integration of biological databases is not trivial [4]. Due to the fact that the completeness of reaction data differs between the databases, it becomes important to combine the available reaction information of the used source databases in form of an integrated reaction database.

So a combination will lead to more complete and reliable metabolic networks. Therefore it is necessary to find identical reactions between the recognized databases. As different compound names and compound IDs, as well as reaction IDs, are in use within the described biochemical reactions a comparison is far from straightforward.

\footnotetext{
* Correspondence: D.Schomburg@tu-bs.de

† Contributed equally

Department of Bioinformatics and Biochemistry, Institute for Biochemistry and Biotechnology, Technische Universität Braunschweig, Langer Kamp 19 B, 38106 Braunschweig, Germany
}

(c) 2011 Lang et al; licensee BioMed Central Ltd. This is an Open Access article distributed under the terms of the Creative Commons

A major obstacle results from the use of generic compound names, e.g. 'an aldehyde' or 'an alcohol'. Furthermore some reactions even occur in the same database twice with different reaction IDs.

Integrated databases exist for diverse biological topics. The TRANSPATH ${ }^{\mathbb{R}}$ database for example is an integrated database which deals with signal transduction information [5]. As an example for an integrated metabolic database system the database BioSilico can be mentioned here [6]. For creation of this database, information of the metabolic databases KEGG, ENZYME [7], EcoCyc [8], and MetaCyc was combined, the latter two building parts of BioCyc [9]. The database BioSilico includes information on enzymes, biochemical compounds, and reactions. Radrich et al. [10] provide a semi-automated tool for the process of genome-scale network reconstruction demonstrated on the basis of data for Arabidopsis thaliana. Their integrated data set is built on the two sources KEGG and AraCyc [11]. Furthermore a reaction database on human biological pathways and processes named Reactome [12] exists as well as an annotated reaction database called Rhea [13], basically a modified version of 
the reactions defined in the $I U B M B$ enzyme list [14]. A collection of biochemical reactions and pathways in printed form contains the book Biochemical Pathways: An Atlas of Biochemistry and Molecular Biology [15].

\section{Methods}

In this work information from the biological databases BRENDA [1], KEGG [2], and MetaCyc [3] was used (May 2011). Reaction comparisons were done by an in silico approach in which two steps, first a comparison of reactant structures using InChIs (linearized chemical structure descriptors [16]) and, second, a compound name comparison (incl. synonyms), were combined. An InChI structure coding was generated based on an original $\mathrm{Mol}$ file (contains molecular structure information [17]) by using a special converting tool (InChI version 1 (software version 1.03) for Standard and Non-Standard InChI/ InChIKey [18]). By using only relevant layers of an selfgenerated InChI, a higher matching rate was achieved. For this purpose we dropped the $I n C h I$ layers dependent on the ionisation state so that e.g. acetic acid and the acetate ion were considered to be the same compound. Reactions without $E C$ numbers were included as well as those reactions with incomplete $E C$ numbers. Spontaneous reactions without $E C$ number were labelled $S P O N$ TANEOUS. Before the comparison, the compounds water $\left(\mathrm{H}_{2} \mathrm{O}\right)$ and proton $\left(\mathrm{H}^{+}\right)$were removed from the reactions. Additionally, a stoichiometry check was executed. This information was added as attribute to the reactions in the database as a quality measure. Stoichiometrically imbalanced reactions were marked as incomplete in the column Stoichiometry, except in cases where only a proton or water is missing. In two supplemental columns the incomplete cases are differentiated into Missing Substrate and Missing Product.

For the compound name based comparison step all found synonyms were used as well as generated ' $D A Y$ LIGHT names' (Chemical Information Systems, Inc. [19]). We applied a special conversion that removed most of the common sources of differences in equivalent compound names like hyphens, parentheses, etc. Most of the special characters, except ' + ' and apostrophe ('), were deleted. For identifying common reactions, all available synonyms and 'DAYLIGHT names' (see above) of the compounds are included in form of a link table containing assigned compound IDs. Where possible, $K E G G$ glycan IDs ( $G$ number) were exchanged by their corresponding compound IDs ( $C$ number). Reactions with $\mathrm{NAD}(\mathrm{P}) / \mathrm{H}(B R E N D A)$ and NADP/H_OR_NO_P $($ MetaCyc) were split into two reactions, one with $\mathrm{NADH}$, the other with NADPH. The reaction ID of the form without phosphate was labelled as the original but with _WOP (= With $\underline{\text { Out }}$ Phosphate) at the end.
Data download, storage, and comparison was realized by $C++$ as well as Python scripts and embedded MySQL statements. By executing a cron-job in regular time points, the information about metabolites, enzymes, reactions, Molfiles, and InChIs was downloaded from the source databases and so kept up to date automatically.

The access to the integrated database is possible via the link to $B K M$-react [20], Figure $1 \mathrm{~A}$, or via the $B R E N D A$ website, making use of the BRENDA query engine. Figure $1 \mathrm{~B}$ illustrates the access to the integrated non-redundant reaction database [21] $\rightarrow$ Reaction $\&$ Specificity $\rightarrow$ Biochemicals Reactions Aligned (see arrow). Parameters for doing queries are presented in Figure $2 \mathrm{~A}$ for the reaction table. Figure $2 \mathrm{~B}$ shows an example for a query result. The downloadable content of the database consists of three tables, containing the compared reactions, the according compounds as well as a link table connecting both with each other.

\section{Results and discussion}

The combined database contains a unique list of reactions that occur in any of the compared databases BRENDA [1], KEGG [2], and MetaCyc [3] and the associations between equivalent reactions. Additionally these reactions are assigned to KEGG and MetaCyc pathways. Table 1 lists the data used for the comparison. The largest number of reactions originates from the BRENDA database, followed by MetaCyc, and KEGG.

A significantly improved matching of reactions was achieved by removing the compounds $\mathrm{H}^{+}$and water $\left(\mathrm{H}_{2} \mathrm{O}\right)$ from the reactions before comparing them because the reactions in the databases are not always stoichiometrically balanced. The order of executing first the $\mathrm{InChI}$ comparison followed by the name comparison was chosen because identical synonyms may occur for different compounds. To rely on synonyms could therefore result in incorrect links. By using the reverse order more false positive matchings would appear.

One of the difficulties in the comparison consists in the - sometimes implied - stereochemistry not given in the compound name. Whereas cases like "alanine" being used for " $L$-alanine" are obviously to be expected, sometimes things become more complicated. For example, in BRENDA and MetaCyc beta-stereochemistry is implied for $C 5$ of $D$-fructose-1,6-bisphosphate, being the major stereoisomer (see Figure 3A and 3B), the KEGG database includes in fact two different reactions, one with betastereochemistry at $\mathrm{C} 5$, the other with undefined stereochemistry (see Figure 4A and 4B) where pathway information is only assigned to the reaction with the full stereochemistry. In general metabolites with complete stereochemistry are favored in BKM-react. 


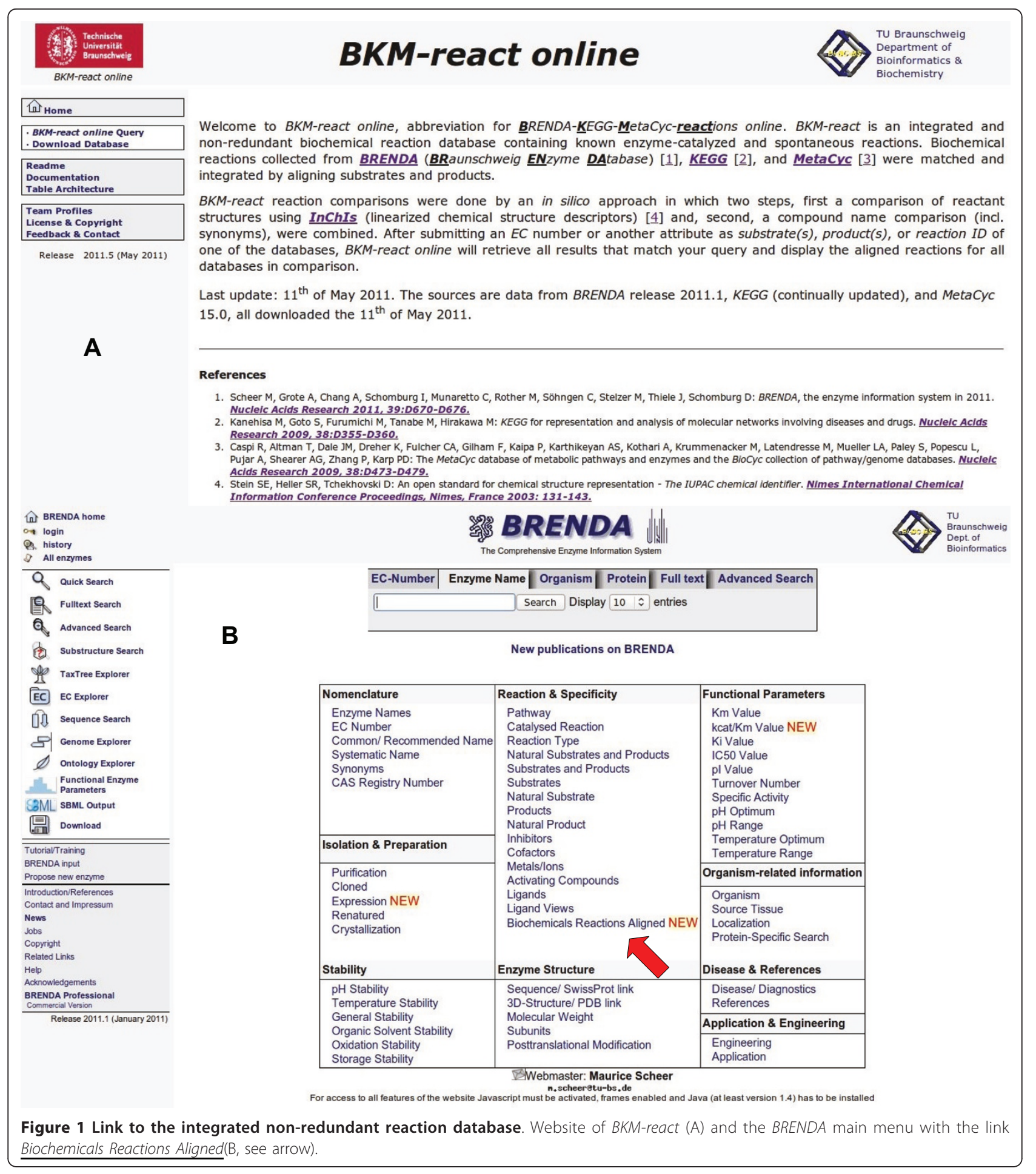

If no structural information is available, reactions are allowed to match by name comparison.

This example shows a general problem in biochemical compound name comparison. The large majority of biochemists refer to $S$-alanine just by the name alanine although the name in principle is ambiguous or should be used for the racemate. In most cases we assume that for the standard amino acids the name without stereo-descriptor implicitly means $S$ - (or $L$-, respectively). This holds true also for some other compound names where the stereo-descriptor is implicitly given. A related problem occurs at positions where the 


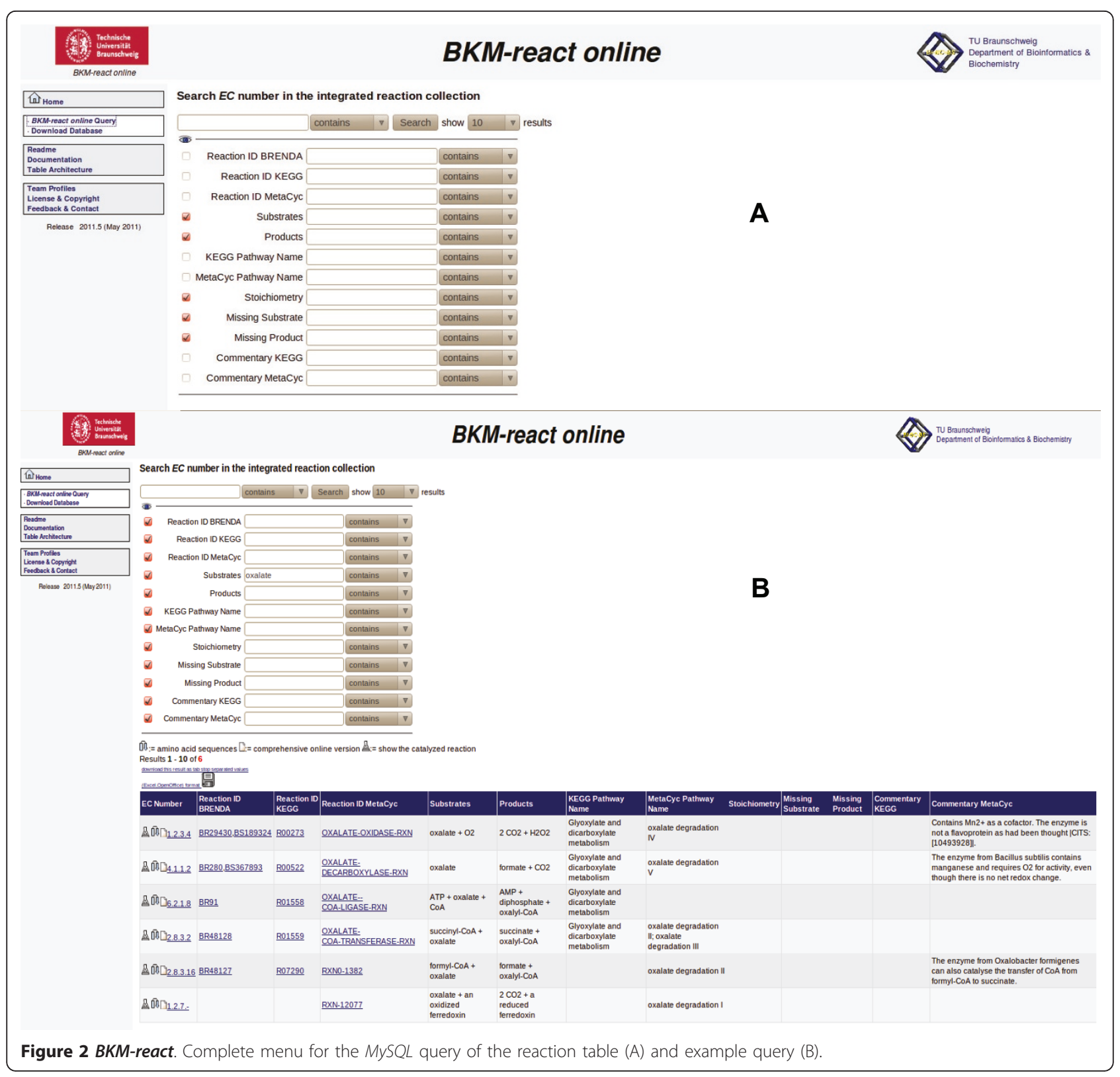

stereochemistry is ambiguous like in the case of $\mathrm{C} 1$ of $D$-glucose. In some cases the stereochemistry for this position is undefined in the Molfiles [17], in others the more stable form (e.g. beta in the case of glucose) is used and defined.
Although all three databases offer their own InChIs, they are not directly comparable because KEGG uses the non-standard form of an InChI, whereby MetaCyc and BRENDA use the standard InChI format. So for a standardized comparison it is necessary to use self-generated

Table 1 Overview reaction sources and data

\begin{tabular}{lcccccccc}
\hline & $\begin{array}{c}\text { Different } \\
\text { EC numbers }\end{array}$ & $\begin{array}{c}\text { Incomplete } \\
\text { EC numbers }\end{array}$ & Reaction IDs & $\begin{array}{c}\text { Reaction IDs } \\
\text { without } E C \text { number }\end{array}$ & Compound IDs & Synonyms & Molfiles & InCh/s \\
\hline KEGG & 3,761 & 122 & 8,452 & 1,288 & 6,522 & 11,597 & 6,327 & 5,416 \\
MetaCyc & 4,159 & 138 & 9,343 & 2,236 & 6,095 & 19,707 & 6,035 & 5,782 \\
BRENDA & 4,425 & 207 & 10,109 & 55 & 9,750 & 20,922 & 9,750 & 5,242 \\
\hline
\end{tabular}

The number of reactions in BRENDA is in fact close to 180,000 . In this case only complete reactions with natural substrates were included. 


\section{Reaction catalyzed by 6-phosphofructokinase (2.7.1.11)}

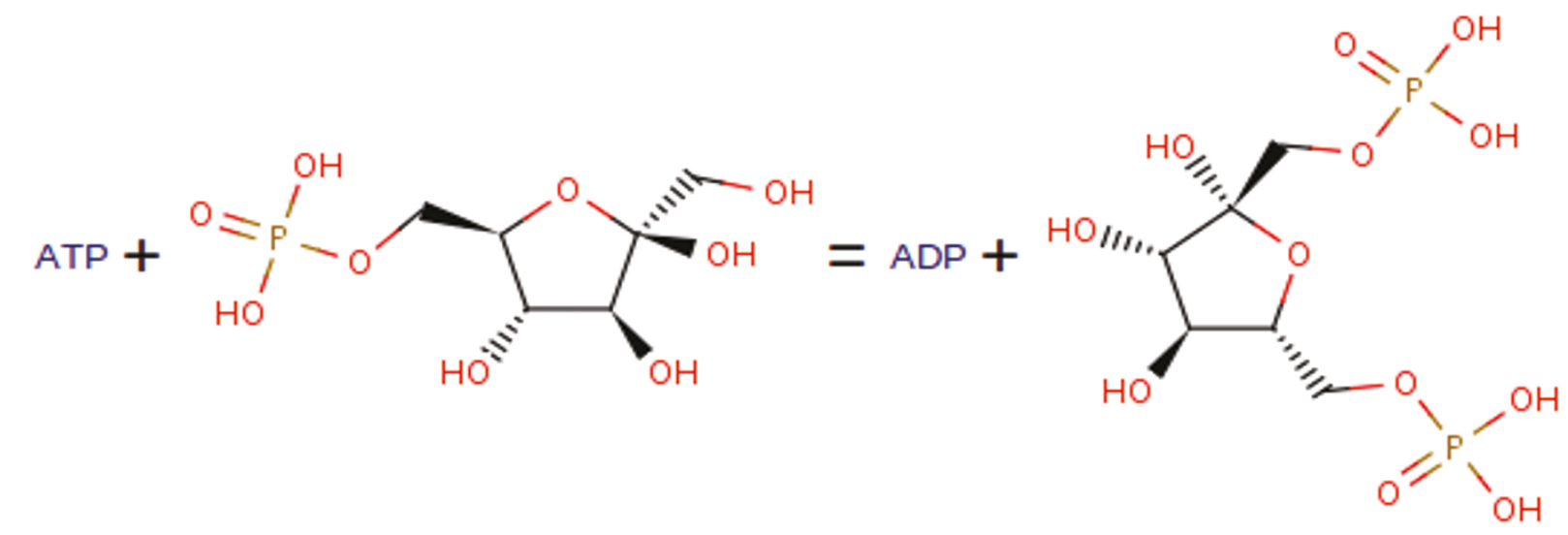

\section{ATP $+\quad$ D-fructose 6-phosphate $\quad=$ ADP + D-fructose 1,6-bisphosphate}

\begin{tabular}{|c|c|c|c|}
\hline ID & CompoundID & Synonym & InChI \\
\hline 9102 & BG_22767 & $\begin{array}{l}\text { D-fructose } \\
1,6 \text {-bisphosphate }\end{array}$ & $\begin{array}{l}\text { InChl=1S/C6H14O12P2 } \\
\text { /c7-4-3(1-16-19(10,11)12)18-6(9,5(4)8)2-17-20(13,14)15 } \\
\text { /h3-5,7-9H,1-2H2,(H2,10,11,12)(H2,13,14,15)/t3-,4-,5+,6- } \\
\text { /m1/s1 }\end{array}$ \\
\hline
\end{tabular}

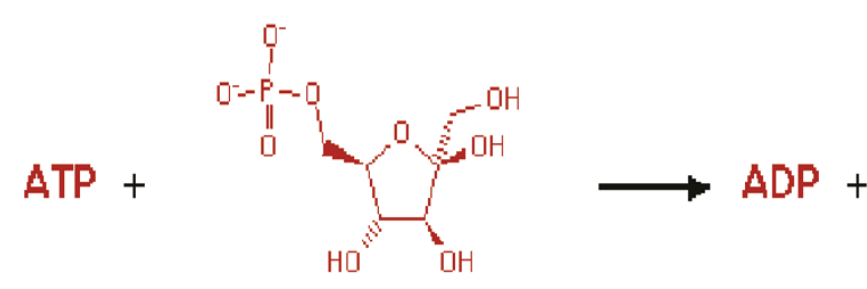<smiles>O=P([O-])(Cl)OC[C@H]1O[C@H](O)[C@@H](O)C1O</smiles>

B

\section{D-fructose-6-phosphate}

\begin{tabular}{|c|c|c|c|}
\hline ID & CompoundID & Synonym & InChI \\
\hline 18225 & FRUCTOSE-6P & $\begin{array}{l}\text { D-fructose- } \\
\text { 6-phosphate }\end{array}$ & $\begin{array}{l}\text { InChl=1S/C6H1309P/c7-2-6(10)5(9)4(8)3(15-6)1-14-16(11,12)13 } \\
\text { /h3-5,7-10H,1-2H2,(H2,11,12,13)/p-2/t3-,4-,5+,6-/m1/s1 }\end{array}$ \\
\hline
\end{tabular}

Figure 3 Screenshots of the databases BRENDA and MetaCyc. These reactions, BRENDA reaction BR47724 and the MetaCyc reaction 6PFRUCTPHOS-RXN are matching the KEGG reaction R04779 (Fig. 4 B) because of the complete InCh/ string for beta-D-Fructose 1,6-bisphosphate even if MetaCyc names it D-fructose-6-phosphate.

InChIs based on Molfiles. For this purpose the official IUPAC converting tool was utilized [18]. A higher matching rate was achieved by using only essential layers (see Methods section) of an InChI string. A drawback is that not for each compound an InChI is available, e.g. for macromolecular reactants or for generic compounds.

A pairwise comparison of reactions revealed a high identity between KEGG \&MetaCyc. About $50 \%$ reactions were equal, out of which most were also found in
BRENDA (Figure 5). Between MetaCyc \&BRENDA 3,174 reactions were identified to be equal. Comparing $K E G G$ $\& B R E N D A$, even more reactions $(3,617)$ could be assigned to each other.

Table 2 shows the assignment of diverse reactions between the databases which are equal. There are examples of reactions that have a 1:n relation because of redundant reactions occurring within the same database. In $K E G G$ for example, metabolites are differentiated into 


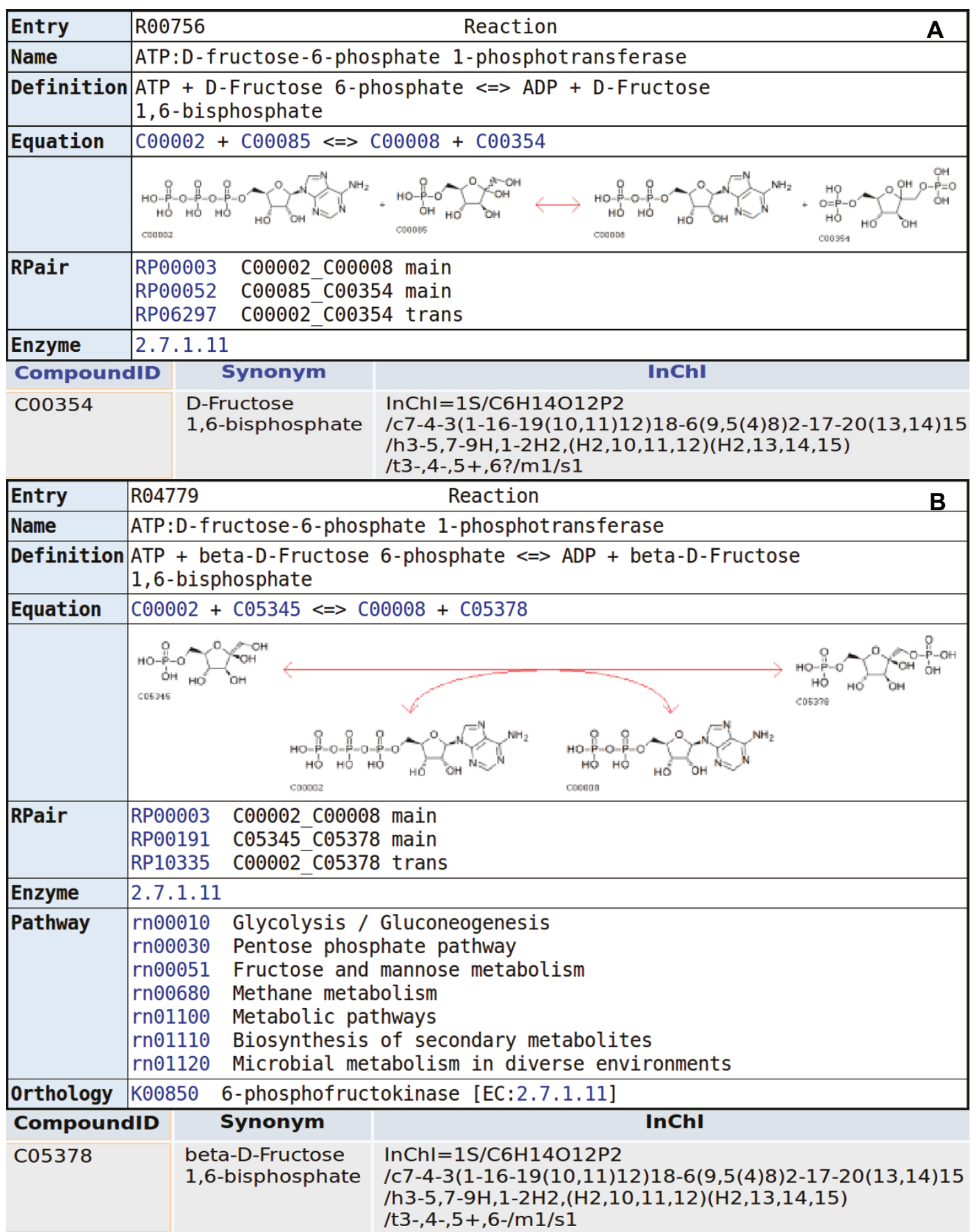

Figure 4 Screenshots of the database KEGG. KEGG R00756 and R04779. The second reaction is the preferred one. C04779 possesses the complete $\operatorname{lnChl}$ string and is therefore matched with the more complete described metabolites of the other databases.

glycans and compounds, respectively. This means that identical compounds may get two different IDs, starting with $G$ and $C$. This results in reactions with different reaction IDs (no. 3 in Tab. 2). Sometimes there are synonyms or keto-enol tautomers which describe one reaction in various forms (no. 1 in Tab. 2) or other alternative writing styles (no. 2 in Tab. 2). Further KEGG uses one reaction-ID for the same reaction being catalysed by 


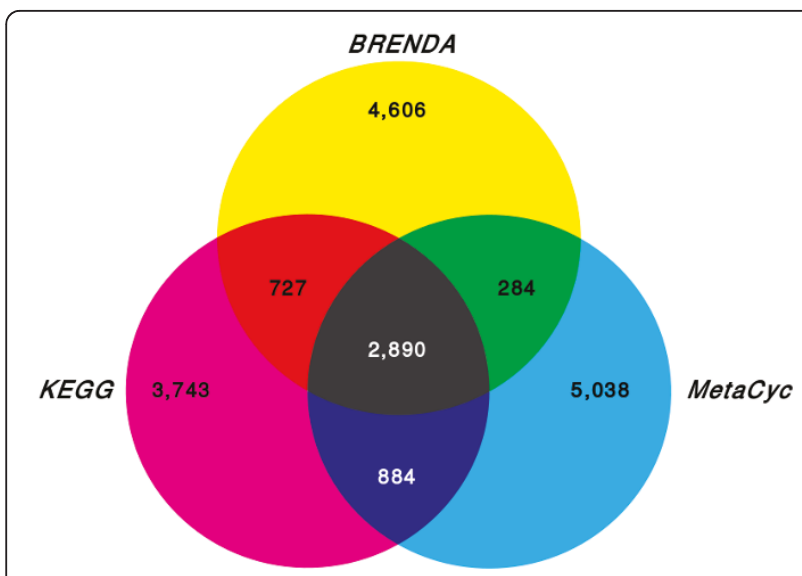

Figure 5 Distribution of unique reactions between the used databases.

enzymes with different $E C$ numbers, whereas MetaCyc often uses different reaction IDs in such cases (no. 1 in Tab. 2).

In Figure 5 the distribution of equal reactions occurring in any of the three databases is illustrated. 2,890 of all reactions are contained in all three databases, corresponding to $34 \%$ of all KEGG reactions, $31 \%$ of all MetaCyc reactions, and 29\% of the included BRENDA reactions, respectively. In the present version of the data set, 3,743 KEGG reactions, 5,038 MetaCyc reactions, and 4,606 BRENDA reactions occur only in the respective database (Figure 5). Altogether the non-redundant reaction database up to now contains 18,172 unique reactions and 20,358 $\mathrm{EC} /$ reaction combinations as some reactions are catalyzed by a number of different enzymes.

In Figure 6 the fraction of all unique reactions belonging to the six main $E C$ classes is shown. The largest fractions belong to $E C$ classes 1 and 2, followed by class 3 . Statistical data about the $E C$ numbers occurring in the

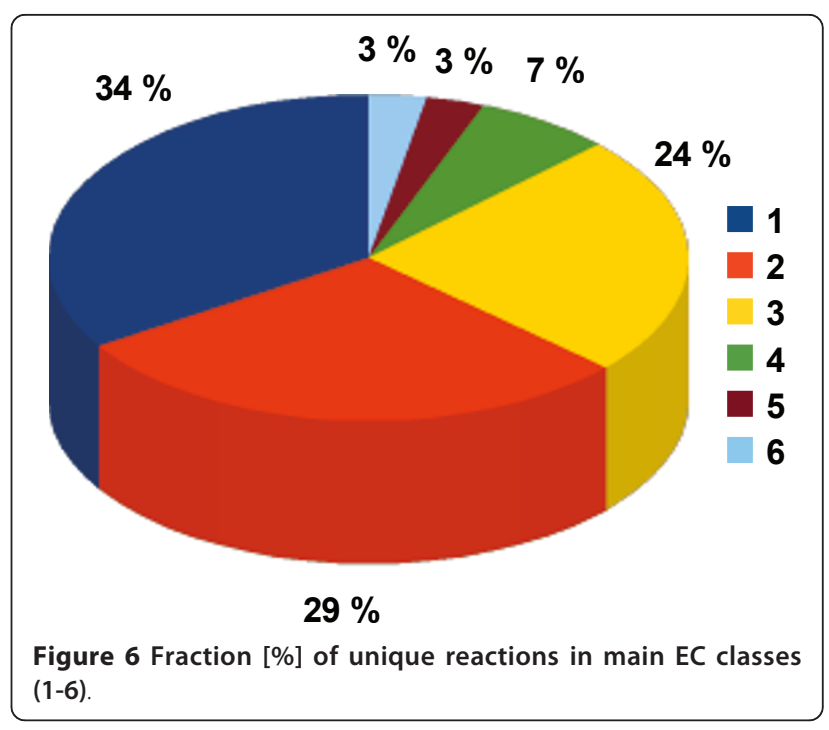

non-redundant reaction database are given in Table 3. Additionally to all EC numbers, complete and incomplete, the latter ones are listed separately. Furthermore it is distinguished between $E C$ numbers representing at least one single reaction or more than one. A detailed look on the $E C$ numbers with the highest number of reactions is given in Table 4 together with the number of reactions.

The only database with a similar goal is BioSilico [6]. One important difference consists of the fact that the assignment of identical reactions in our database is done by an actual comparison of the compounds structure in combination with synonyms whereas in BioSilico, the matching is only a simple assignment of reactions having the same $E C$ number without redundancy check.

The number of reactions in the database described in this paper is far beyond that in BioSilico. Selecting three $E C$ numbers by chance resulted in e.g. EC number 1.14.14.1 $\rightarrow 4$ reactions in BioSilico vs. 116 reactions in

Table 2 Some instructive cases of different forms for identical reactions

\begin{tabular}{|c|c|c|c|c|}
\hline No. & KEGG & MetaCyc & BRENDA & Definition \\
\hline \multirow[t]{4}{*}{1} & $R 04915$ & & & Quinoline-3,4-diol + Oxygen $<=>$ Formylanthranilate + CO \\
\hline & R05719 & & & 3-Hydroxy-1H-quinolin-4-one + Oxygen $<=>$ Formylanthranilate + CO \\
\hline & & 1.13.11.47-RXN & & 3-hydroxy-1H-quinolin-4-one + oxygen $=$ carbon monoxide $+\mathrm{N}$-formylanthranilate \\
\hline & & & $B R 22597$ & 3-hydroxy-1H-quinolin-4-one + $\mathrm{O} 2$ = N- formylanthranilate + $\mathrm{CO}$ \\
\hline \multirow[t]{3}{*}{2} & R00004 & & & Diphosphate $+\mathrm{H} 2 \mathrm{O}<=>2$ Orthophosphate \\
\hline & & INORGPYROPHOSPHAT-RXN & & diphosphate $+\mathrm{H}_{2} \mathrm{O}=2$ phosphate $+\mathrm{H}^{+}$ \\
\hline & & & $B R 22749$ & diphosphate $+\mathrm{H} 2 \mathrm{O}=2$ phosphate \\
\hline \multirow[t]{5}{*}{3} & $R 00010$ & & & alpha, alpha-Trehalose $+\mathrm{H} 2 \mathrm{O}<=>2$ D-Glucose (C01083) \\
\hline & R06103 & & & Trehalose + H2O <=> 2 D-Glucose (G00293) \\
\hline & & TREHALA-RXN & & trehalose $+\mathrm{H} 2 \mathrm{O} \rightarrow 2 \beta$-D-glucose \\
\hline & & & BR15991 & alpha, alpha-trehalose $+\mathrm{H} 2 \mathrm{O}=2 \mathrm{D}$-glucose \\
\hline & & & BS370856 & alpha, alpha-trehalose $+\mathrm{H} 2 \mathrm{O}=$ beta-Dglucose \\
\hline
\end{tabular}


Table 3 Statistics about EC numbers occurring in the integrated non-redundant reaction database

\begin{tabular}{ccc}
\hline $\boldsymbol{E} C$ numbers & Different $\boldsymbol{E C}$ numbers & Incomplete $\boldsymbol{E C}$ numbers \\
\hline in total & 4,288 & 365 \\
with $>1$ reaction & 2,681 & 185 \\
with $>5$ reactions & 561 & 73 \\
with $>10$ reactions & 184 & 49 \\
\hline
\end{tabular}

our reaction database, $E C$ number $2.1 .1 .103 \rightarrow 1$ reaction in BioSilico vs. 4 reactions in our database, 3.1.1.47 $\rightarrow 1$ reaction in BioSilico vs. 12 reactions in our database. The fact that in these examples not even all available KEGG reactions were found in BioSilico indicates that this database is not updated.

Additionally, our reaction database contains the information whether a reaction is stoichiometric incomplete or not. This test is performed before the removal of $\mathrm{H}^{+}$ and $\mathrm{H}_{2} \mathrm{O}$. Non-balanced reactions are labeled in a separate table column. 2,803 out of 18,172 reactions are at present in this category. The labeling allows modelers to select only balanced reactions for the reconstruction of organism-specific models and networks.

The tool of Radrich et al. [10] also includes a stoichiometric evaluation. Their method includes a name comparison where they compare the similarity of compound names. Further they use SMILES strings for a structural comparison. The tool was executed only for Arabidopsis thaliana, so no general comparison could be done. For this purpose the authors combined data of the databases KEGG and AraCyc [11].

\section{Conclusions}

In this work we present an integrated and non-redundant reaction database implementing a combined approach of structure and name based comparison.

The tool, integrated into the $B R E N D A$ [1] query engine but not confined to BRENDA data is offering a novel valuable tool that can be used e.g. for the construction of biological models. The resulting models will be much more complete than if only one of the databases is used as the three biological databases BRENDA, KEGG [2], and MetaCyc [3] complement each other. Regular 6monthly updates of this database will make guarantee actuality.

\section{Availability and requirements}

The integrated and non-redundant reaction database is accessible via $B K M$-react [20] and the website of the BRENDA [1] database: BRENDA website [21] $\rightarrow$ Reaction E Specificity $\rightarrow$ Biochemicals Reactions Aligned (Figure 1). The complete dataset is additionally provided as a CSV-formatted download at the same site. Available is a reaction table, a table with all compounds occurring in the reactions, and an assignment table with the linkage between reactions and compounds.

Table 4 Complete EC numbers with the highest number of reactions

\begin{tabular}{|c|c|c|}
\hline EC number & Enzyme & Number of reactions \\
\hline 1.14 .14 .1 & unspecific monooxygenase & 116 \\
\hline 2.4.1.17 & glucuronosyltransferase & 80 \\
\hline 3.2.1.21 & beta-glucosidase & 74 \\
\hline 1.1.1.100 & 3-oxoacyl-[acyl-carrier-protein] reductase & 55 \\
\hline 3.5.1.4 & amidase & 46 \\
\hline 3.6.3.44 & xenobiotic-transporting ATPase & 46 \\
\hline 3.1.3.16 & phosphoprotein phosphatase & 44 \\
\hline 1.3.1.10 & enoyl-[acyl-carrier-protein] reductase (NADPH, B-specific) & 43 \\
\hline 3.2.1.1 & alpha-amylase & 43 \\
\hline 1.1 .1 .50 & 3alpha-hydroxysteroid dehydrogenase (B-specific) & 42 \\
\hline 2.3.1.41 & beta-ketoacyl-acyl-carrier-protein synthase I & 41 \\
\hline 3.6.1.9 & nucleotide diphosphatase & 39 \\
\hline 1.1.1.1 & alcohol dehydrogenase & 37 \\
\hline 2.3.1.86 & fatty-acyl-CoA synthase & 37 \\
\hline 1.14.13.72 & methylsterol monooxygenase & 36 \\
\hline 1.2 .1 .3 & aldehyde dehydrogenase (NAD $\left.{ }^{+}\right)$ & 34 \\
\hline 1.2.1.5 & aldehyde dehydrogenase $\left[\mathrm{NAD}(\mathrm{P})^{+}\right]$ & 33 \\
\hline 3.2 .1 .24 & alpha-mannosidase & 33 \\
\hline 3.2.1.51 & alpha-L-fucosidase & 33 \\
\hline 1.14.13.8 & flavin-containing monooxygenase & 32 \\
\hline 1.4 .3 .3 & D-amino-acid oxidase & 32 \\
\hline
\end{tabular}

Recommended names of enzymes: source BRENDA database. 


\section{List of abbreviations used}

BRENDA: BRaunschweig ENzyme DAtabase; EC: Enzyme Commission; InChl: IUPAC International Chemical Identifier; IUBMB: International Union of Biochemistry and Molecular Biology; IUPAC: International Uniōn of Pure and Äpplied Chemistry; KEGG: Kyōto Encyclopedia of Genes and Genomes; SMILES: Simplified Molecular Inpūt Line Entry System.

\section{Acknowledgements and funding}

The authors are grateful to Ron Caspi from MetaCyc for help with the implementation of the MetaCyc data, Adam Podstawka for technical support, Maurice Scheer for implementing the webinterface, and René Rex for providing a stoichiometry verification tool. Financial support: European Union (FELICS [22], SLING [23]).

\section{Authors' contributions}

ML and MS executed the data acquisition and implemented the reaction comparison. ML and MS were involved in the construction of the integrated reaction database and the scientific evaluation. DS had the idea to develop the reaction database and supervised the development. ML, MS, and DS wrote the manuscript. All authors read and approved the final manuscript.

\section{Received: 10 January 2011 Accepted: 8 August 2011}

Published: 8 August 2011

\section{References}

1. Scheer M, Grote A, Chang A, Schomburg I, Munaretto C, Rother M, Söhngen C, Stelzer M, Thiele J, Schomburg D: BRENDA, the enzyme information system in 2011. Nucleic Acids Research 2011, 39:D670-D676.

2. Kanehisa M, Goto S, Furumichi M, Tanabe M, Hirakawa M: KEGG for representation and analysis of molecular networks involving diseases and drugs. Nucleic Acids Research 2009, 38:D355-D360.

3. Caspi R, Altman T, Dale JM, Dreher K, Fulcher CA, Gilham F, Kaipa P, Karthikeyan AS, Kothari A, Krummenacker M, Latendresse M, Mueller LA, Paley S, Popescu L, Pujar A, Shearer AG, Zhang P, Karp PD: The MetaCyc database of metabolic pathways and enzymes and the BioCyc collection of pathway/genome databases. Nucleic Acids Research 2009, 38:D473-D479.

4. Stein LD: Integrating biological databases. Nat Rev Genet 2003, 4:337-345.

5. Krull M, Voss N, Choi C, Pistor S, Potapov A, Wingender E: TRANSPATH ${ }^{\circ}$ an integrated database on signal transduction and a tool for array analysis. Nucleic Acids Research 2003, 31:97-100

6. Hou BK, Kim JS, Jun JH, Lee D-Y, Kim YW, Chae S, Roh M, In Y-H, Lee SY: BioSilico: an integrated metabolic database system. Bioinformatics 2004, 20:3270-3272.

7. ExPASy - ENZYME. [http://www.expasy.org/enzyme/]

8. EcoCyc: Encyclopedia of Escherichia coli K-12 Genes and Metabolism. [http://ecocyc.org/].

9. BioCyc Home. [http://biocyc.org/].

10. Radrich K, Tsuruoka Y, Dobson P, Gevorgyan A, Swainston N, Baart G, Schwartz J-M: Integration of metabolic databases for the reconstruction of genome-scale metabolic networks. BMC Systems Biology 2010, 4:114.

11. TAIR - AraCyc. [http://www.arabidopsis.org/biocyc/index.jsp].

12. Matthews L, Gopinath $G$, Gillespie M, Caudy M, Croft D, de Bono B, Garapati P, Hemish J, Hermjakob H, Jassal B, Kanapin A, Lewis S, Mahajan S, May B, Schmidt E, Vastrik I, Wu G, Birney E, Stein L, D'Eustachio P: Reactome knowledgebase of human biological pathways and processes. Nucleic Acids Research 2009, 37:D619-D622.

13. Rhea - Annotated reactions database. [http://www.ebi.ac.uk/rhea//home. xhtmllt].

14. IUBMB Nomenclature Home Page. [http://www.chem.qmul.ac.uk/iubmb/].

15. Michal G, Schomburg D: Biochemical Pathways: An Atlas of Biochemistry and Molecular. Biology. 2 edition. Wiley-Interscience; 2011.

16. Stein $S E$, Heller SR, Tchekhovski D: An open standard for chemical structure representation-the IUPAC chemical identifier. Nimes International Chemical Information Conference Proceedings, Nimes, France 2003, 131-143

17. Dalby A, Nourse JG, Hounshell WD, Gushurst AKI, Grier DL, Leland BA, Laufer J: Description of several chemical structure file formats used by computer programs developed at Molecular Design Limited. Journal of Chemical Information and Computer Sciences 1992, 32:244-255.

18. International Union of Pure and Applied Chemistry. [http://www.iupac. org/inchi/download/index.html].
19. Daylight. [http://www.daylight.com/].

20. BKM-react online. [http://bkm-react.tu-bs.de/].

21. Enzyme Database - BRENDA. [http://www.brenda-enzymes.org/]

22. FELICS | FELICS. [http://www.felics.org].

23. SLING | sling-fp7.org. [http://www.Sling-fp7.org/].

\section{doi:10.1186/1471-2091-12-42}

Cite this article as: Lang et al: BKM-react, an integrated biochemical reaction database. BMC Biochemistry 2011 12:42.

\section{Submit your next manuscript to BioMed Central and take full advantage of:}

- Convenient online submission

- Thorough peer review

- No space constraints or color figure charges

- Immediate publication on acceptance

- Inclusion in PubMed, CAS, Scopus and Google Scholar

- Research which is freely available for redistribution

Submit your manuscript at www.biomedcentral.com/submit
C Biomed Central 